Article

\title{
An Easy-Implemented On-Chip Waveguide Coupled Single Photon Source Based on Self-Assembled Quantum Dots Membrane
}

\author{
Ping Jiang ${ }^{1, *} \mathrm{Na} \mathrm{Ma}^{1}$, Peng Liu ${ }^{1}$, Wenxuan $\mathrm{Wu}^{1}{ }^{1}$ and Kai Zhang ${ }^{2}$ \\ 1 College of Science, China University of Petroleum, Changjiang West Road 66, Qingdao 266580, China; \\ s20090093@s.upc.edu.cn (N.M.); liupeng@upc.edu.cn (P.L.); 1909040113@s.upc.edu.cn (W.W.) \\ 2 College of Geosciences, China University of Petroleum, Changjiang West Road 66, Qingdao 266580, China; \\ zhksam@163.com \\ * Correspondence: pjiang@upc.edu.cn
}

check for

updates

Citation: Jiang, P.; Ma, N.; Liu, P.;

Wu, W.; Zhang, K. An

Easy-Implemented On-Chip

Waveguide Coupled Single Photon

Source Based on Self-Assembled

Quantum Dots Membrane. Appl. Sci.

2021, 11, 695. https://doi.org/

10.3390/app11020695

Received: 7 December 2020

Accepted: 8 January 2021

Published: 13 January 2021

Publisher's Note: MDPI stays neutral with regard to jurisdictional clai$\mathrm{ms}$ in published maps and institutional affiliations.

Copyright: (C) 2021 by the authors. Licensee MDPI, Basel, Switzerland. This article is an open access article distributed under the terms and conditions of the Creative Commons Attribution (CC BY) license (https:// creativecommons.org/licenses/by/ $4.0 /)$.
Featured Application: This work has an application in realizing on-chip quantum photonic circuits with integrated quantum emitters for quantum computation or quantum communication.

\begin{abstract}
In recent years, many groups and institutions have been committed to the research of integrated quantum photonic circuit technologies, of which the key components are waveguide coupled single photon sources. In this study, we propose an on-chip waveguide-coupled single photon source that is easily implemented as the waveguide is directly made from the quantum dot membrane. In order to scatter light out of the on-chip waveguide plane into the detection apparatus, grating output couplers are made at both ends of the waveguide. The photon statistics of the on-chip photon source were investigated by second-order correlation function $\mathrm{g}^{(2)}(\tau)$ measurements using a Hanbury Brown and Twiss interferometer. From the spectra and cross-correlation experiments by collecting emission at the point of quantum dot and out coupler, the emitting of single photons from the same quantum dot and propagating via the waveguide to the out couplers was confirmed. These results show that we have achieved an on-chip single photon source that is easily implemented and easily integrated into quantum photonic circuits.
\end{abstract}

Keywords: quantum photonic circuit; single photon source; waveguide coupled; self-assembled quantum dot

\section{Introduction}

In the research fields of quantum information and quantum communications, single photons have attracted significant attention as they are fundamental elements for current quantum technologies [1-3]. Currently, quantum technologies are undergoing a revolution as the research on integrated quantum photonic circuits (QPCs) opens up exciting opportunities to implement complex quantum algorithms on single chips. For realization of QPCs, single photon sources and processing devices as well as photon detectors have to be combined together on single chips. The single photon sources need to be integrated into quantum photonic circuits to realize on-chip systems by directly coupling with nanophotonic devices via low loss waveguides. Therefore, an efficient coupling of single photon sources with waveguides remains an attracting issue, as the waveguide coupled single photon source is the key component of a QPC. Many efforts to place single photon sources in or on waveguides have been reported based on single photons generated from the $\mathrm{NV}$-center in nano-diamonds [4], quantum dots (QDs) [5,6], and single walled carbon nanotubes [7].

Compared with other kinds of single photon emitters, self-assembled quantum dots are very attractive candidates as they meet the requirements of high extraction efficiency, 
narrow emission linewidth, high single photon emission purity, stability, and so on. Integration of QDs with circuit elements has also been demonstrated by structuring the semiconductor material [8]. Among them, semiconductor III-V QDs are an ideal choice for implementing hybrid integrated QPCs for their high performance of emitting nearideal single photons with ultrahigh brightness and indistinguishability, which also can be site-controlled and compatible with semiconductor manufacturing technology [9-11]. Embedding of III-V QDs into integrated quantum circuits has been realized by various approaches [12-14]. In these works, special methods or techniques are used to integrate III-V QDs on photonic chip such as wafer bonding, the micromanipulation method, or by the "pick-place" technique $[12,14,15]$, which makes the implementation not as easy. Moreover, as the development of highly-integrated systems that can solve complex problems mostly depends on the system efficiency, photon flux, passive losses, and detection efficiency should all be optimized while realizing quantum photonic circuits. Although much effort is being devoted to improving the external source coupling efficiency $[16,17]$, such approaches that aim at probing individual devices on a chip do not allow for highly complex circuits. A scalable alternative is to create on-chip sources that are directly integrated into the passive waveguide network $[13,18]$. Resonance fluorescence (RF) from QD coupled into single-mode waveguide has been reported [19]. More approaches are also demonstrated to integrate a quantum emitter with on-chip beam splitters [20-22]. For the latest research, the control of chiral effects in deterministically fabricated QD-waveguide systems and Purcell-enhanced single-photon source based on a quantum dot coupled to a robust on-chip integrated resonator have been reported $[23,24]$.

In this paper, we designed and implemented an on-chip single photon source based on a semiconductor III-V quantum dot, which is directly coupled into the waveguide. Via output couplers at both ends of the waveguide, non-classical light is extracted. The quantum dot membrane that contains emitters is released from bulk crystal and picked up by a new substrate such as $\mathrm{SiO}_{2}$. Then, the waveguide and output coupler structures are directly made from the QD membrane. This greatly reduces the difficulty of the fabrication process for integrating a single source into an on-chip circuit. The on-chip single photon source is directly integrated into the passive waveguides, which will dramatically improve the source coupling efficiency. By numerical analysis, a coupling efficiency of more than 76 percent from the single emitter to the waveguide was achieved. By collecting the emission in the exciton line both from the quantum dot and the output coupler into a Hanbury Brown and Twiss (HBT) interferometer, the second-order cross correlation function $\mathrm{g}^{(2)}(\tau)$ was measured to prove the single photon nature of our waveguide coupled on-chip photon source. Our approach provides an easy way to implement quantum photonic devices integrated with a single photon source for on-chip quantum information processing.

\section{Design and Method}

\subsection{Design}

A design scheme for the on-chip waveguide coupled single photon sources is illustrated in Figure 1. A GaAs QD embedded in a single mode AlGaAs waveguide emits single photons by off resonant excitation of a $532 \mathrm{~nm}$ continuous wave laser. Then, the single photons transmit into and propagate $\sim 2.5 \mu \mathrm{m}$ along the single-mode waveguide. Numerical simulation results show that the transmission efficiency from the QD single emitter to the waveguide with a width of $300 \mathrm{~nm}$ and height of $85 \mathrm{~nm}$ was about $76 \%(38 \%$ for each propagation direction). By adding grating output couplers consisting of $\lambda /(2 n)$ pitch grating $[19,25,26]$ to both ends of the waveguide, emissions were scattered out of the on-chip plane into the detection apparatus. For a designed grating coupler with a period of $400 \mathrm{~nm}$ and ring width of $110 \mathrm{~nm}$, simulated out coupling efficiency was about $40 \%$, similar to other reports. The waveguide and out coupler structures are all directly fabricated from the QD membrane placed on a $\mathrm{SiO}_{2}$ substrate. 


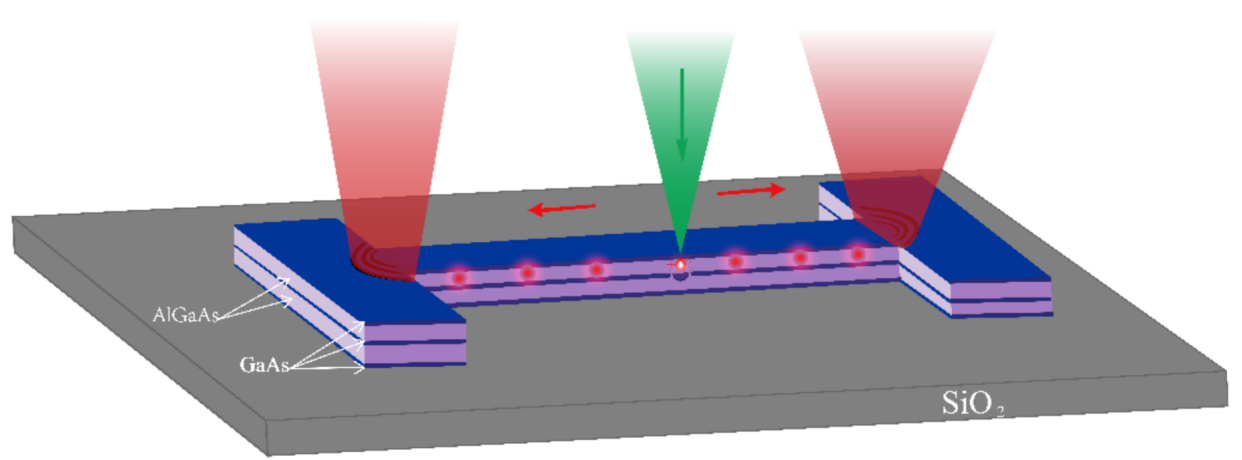

Figure 1. Design of the on-chip waveguide coupled single photon source.

\subsection{Materials}

We realized our design by using a kind of self-assembled quantum dot material of GaAs QDs embedded in an AlGaAs matrix, which was grown on a GaAs substrate by the molecular beam epitaxy (MBE) method [27]. The QDs were fabricated by etching nanoholes directly in the AlGaAs surface using $\mathrm{Al}$ droplets without supplying arsenic. Then, by filling $2 \mathrm{~nm} \mathrm{GaAs}$ into the holes and capping the filled holes with AlGaAs material, QDs are formed [28]. The top layer of the material containing GaAs QD emitters can be released from the bulk GaAs substrate by etching away a sacrificial layer of AlAs that is grown on the substrate in advance. Then, the QD membrane is transferred to a new substrate. Here, a Si substrate with a $400 \mathrm{~nm} \mathrm{SiO}$ on top was used in our experiment. It should be noted that the new substrate can be other materials depending on the demand of integration photonic circuits. A waveguide with grating output couplers at both ends was directly fabricated from the quantum dot membrane by the fabrication process of electron beam lithography (EBL) and reactive ion etch (RIE).

\subsection{Optical Setup}

Optical measurements were performed using a confocal microscope system with the sample cooled down to $10 \mathrm{~K}$. The excitation source was a $532 \mathrm{~nm}$ continuous wave laser. An objective of NA 0.7 was used to both excite the photoluminescence (PL) of the QD and collect the PL from the QD and out-couplers (shown as the red spots in the structure). A sketch of the detection part of the optical setup is shown in Figure 2. The spectra of the QD photoluminescence was recorded by a grating spectrometer equipped with a charge coupled device (CCD). This spectrometer was also used as a monochromator by changing mode to guide the light out of the exit slit. The light spots from the quantum dot and the output couplers were dispersed (shown as the inset picture). Then, only the photons corresponding to the exciton line of the QD moved into a HBT setup for the correlation function measurements. The HBT setup consisted of a 50:50 prism beam splitter, two identical single photon avalanche diodes (SPADs), and a single photon counting module. In addition, a pair of cylindrical mirrors were set before the beam splitter to separate the light spots from the QD and output couplers as much as possible. Then, two SPADs were adjusted to different heights to receive photons from different positions. The time correlated single photon counting module connected to the two SPADs measures the arrival times between photons on the two detectors and produces the second order correlation function. 


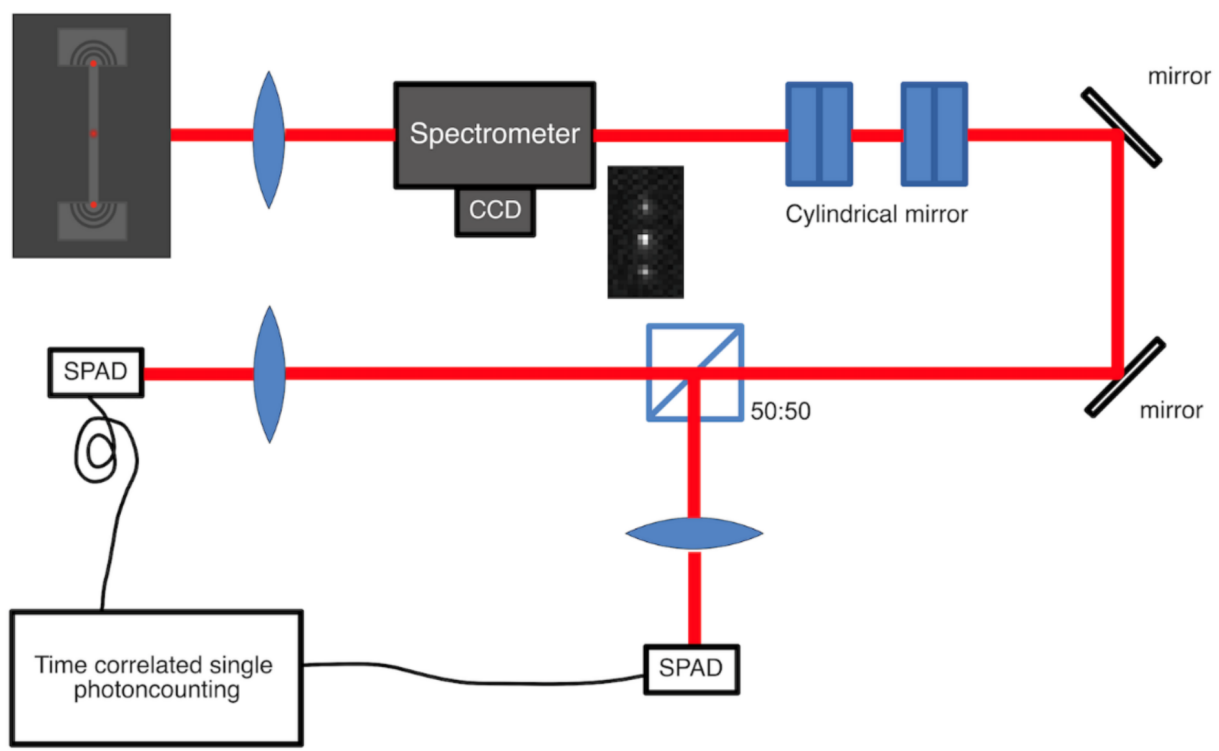

Figure 2. Schematic of the optical detection setup.

\section{Results and Discussion}

\subsection{Photoluminescence Spectra}

Photoluminescence spectra were collected separately from the emitting QD and the grating output couplers (vertically above the QD and output coupler). As shown in Figure 3, the exciton peak (neutral exciton X) of the QD was at $1.5722 \mathrm{eV}$, which was excited by a $532 \mathrm{~nm}$ continuous wave laser with a power of $4.7 \mu \mathrm{W}$. The spectra exhibited exactly the same features, not only in terms of the spectra position, but also the width of the sharp lines, which confirms that the emission from the grating output coupler comes from the emitting quantum dot. The full width at half-maximum for the emission peak collected both from the emitting QD and the grating output coupler were almost the same with a value of $180 \mu \mathrm{eV}$. The photoluminescence intensity collected at the point of the output coupler, which was estimated to be larger by the finite difference time domain (FDTD) simulated results, was actually less than that collected at the point of QD. As above-mentioned, the coupling efficiency from the single emitter to the waveguides was $38 \%$ for each direction and the out coupling efficiency of the grating couplers to scatter emission out of the on-chip plane into detection apparatus was about $40 \%$. Then, about $15.2 \%$ of the photoluminescence from the QD was collected from the point of the grating coupler. The simulated collection efficiency directly from the QD was $9.1 \%$, but from the experimental result, the photoluminescence intensity collected at the output coupler was about $2 / 3$ of the photoluminescence intensity collected at the QD. A reason for this could be the out-coupler's emission efficiency and the waveguide transmission efficiency was not as large as the simulation result as the real structure was not as perfectly as designed when fabricated. In addition, permittivity of the real AlGaAs material used for our sample may be slightly different from the value used for simulation from the literature [29], while a sight deviation of the imaginary part of the refractive index of AlGaAs will increase the waveguide loss drastically. Therefore, an optimized design needs to be carried out to improve the transmission and out coupling efficiency in order to obtain more photon counts. 


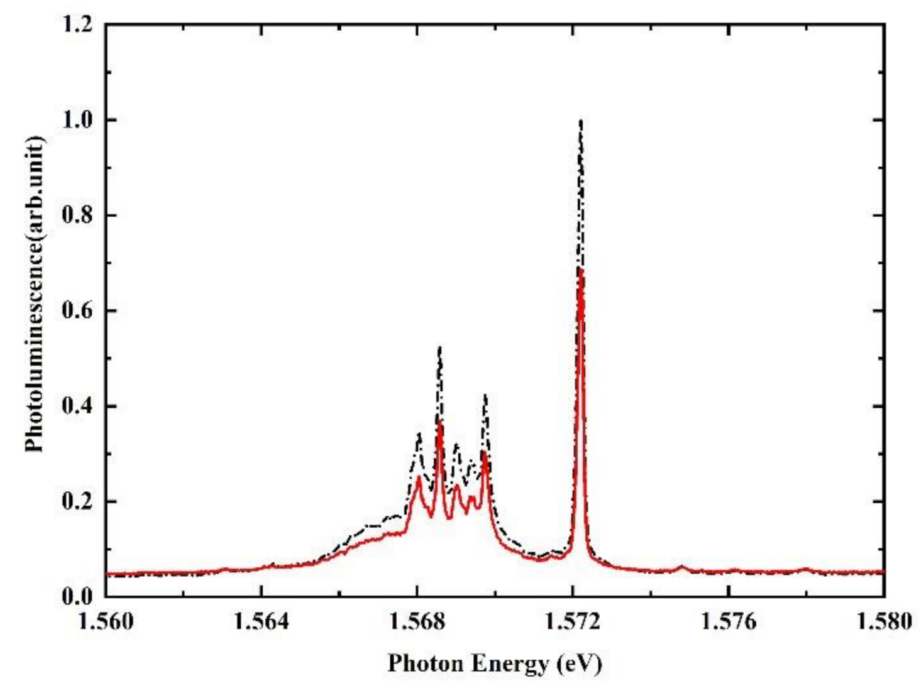

Figure 3. Spectra collected at positions of the quantum dot (black dash line) and out-coupler at the end of the waveguide (red line).

\subsection{Single-Photon Nature}

The HBT experiment setup was used to verify the single photon nature of our waveguide coupled on-chip source. The second-order correlation function $\mathrm{g}^{(2)}(\tau)$ was measured both from the emission at the position of the quantum dot (a) and at the point of output coupler (b). Caused by low coupling efficiency, the count rate is not very high, only about 2000 counts per second. In order to achieve reasonable photon count rates, the power was set to have the strongest exciton line. The signal-to background ratio decreased leading to a diminished contrast of the antibunching with increasing power levels [30]. Depicted in Figure 4, from a fit to the raw data, we can see that $\mathrm{g}^{(2)}(\tau=0)$ was clearly below 0.1 in the configurations and revealed excellent single photon behavior. As shown in Figure 4c, the second-order cross correlation function $\mathrm{g}^{(2)}(\tau)$ between the emission from the QD and the output coupler was also measured, which proved that photons collected at the end of the waveguide originated from the QD. The results unambiguously proved that a single photon emission from GaAs QD was successfully generated and routed along the on-chip waveguide with preservation the single photon behavior. To clarify, we chose the output coupler with a higher emission photon counts for the measurements.

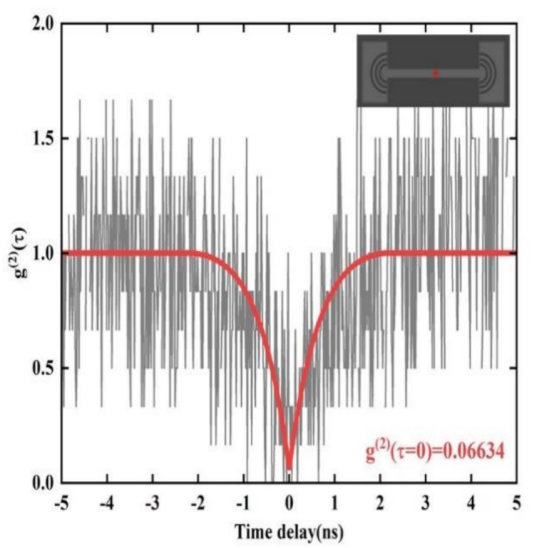

(a)

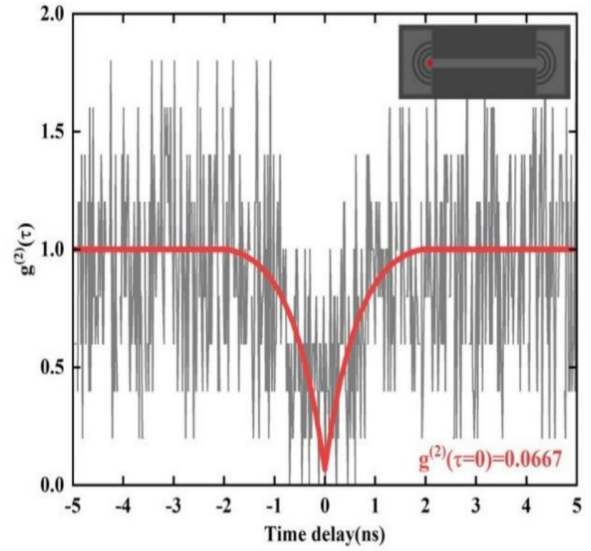

(b)

Figure 4. Cont. 


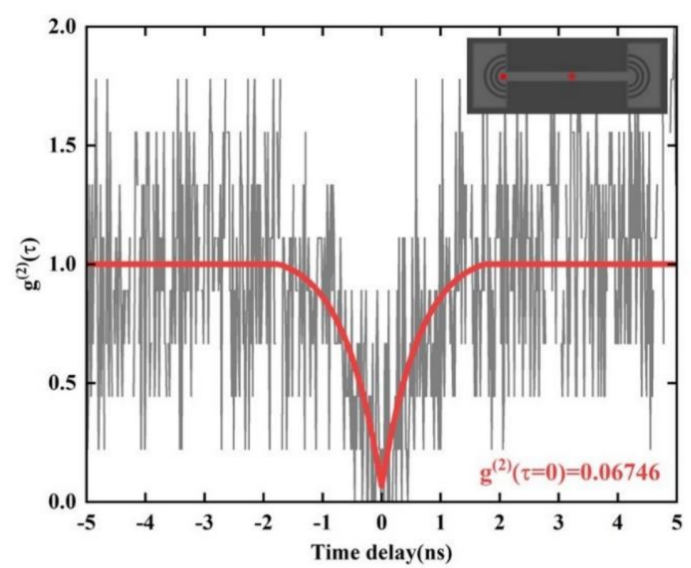

(c)

Figure 4. Second-order correlation measurements of the photons collected from (a) the quantum dot (QD) position, (b) the output coupler position, (c) the QD position, and the output coupler position, shown as red spots on the structure.

\section{Discussion}

A simple model of on-chip single photon source with a self-assembled quantum dot directly coupled with on-chip waveguide has been demonstrated in this paper, which is a key step toward realizing QD-based quantum photonic circuits. The waveguide is directly made from the QD membrane, which greatly reduces the difficulty of the fabrication process for integrating a single source into an on-chip circuit. Based on our approach, a single photon source can be easily coupled with other elements of quantum photonic networks, just as beam splitters, directional couplers, waveguide filters, and so on. Due to the flexible selectivity of the new substrate, the single photon source can be compatible with a variety of quantum circuits. Moreover, multiple quantum dots can be coupled via waveguides to achieve more functions [13,31-34]. Then, more complex quantum photonic circuits will be developed in the future. However, for our design, the QD transitions should be tuned into resonance first, which may be possible by electrical modulation $[35,36]$.

\section{Conclusions}

In summary, we have demonstrated an on-chip waveguide coupled single photon source based on the self-assembled QD membrane. It is easy to implement as the waveguide is directly fabricated in the QD membrane. Based on our approach, more complex elements of quantum photonic circuits can be developed. In the near future, we will focus on improving the photon flux to enable ultrabright on-chip single photon sources with high indistinguishability.

\section{Patents}

A patent application is being filed.

Author Contributions: Conceptualization, P.J.; Methodology, P.J.; Validation, P.J., N.M. and P.L.; Formal analysis, P.J.; Investigation, P.J. and P.L.; Data Curation N.M. and W.W.; Writing-original draft preparation, P.J. and N.M.; Writing-review and editing, K.Z.; Supervision, P.J. All authors have read and agreed to the published version of the manuscript.

Funding: This research was funded by Central University Basic Research Fund (grant number 19CX02056A) and the Major National Science and Technology Projects, (grant number 2017ZX05032-003-002).

Data Availability Statement: Data is contained within the article.

Acknowledgments: We additionally thank Jiaxiang Zhang for the QD material and for help with our work.

Conflicts of Interest: The authors declare no conflict of interest. 


\section{References}

1. Knill, E.; Laflamme, R.; Milburn, G.J. A scheme for efficient quantum computation with linear optics. Nature 2001, 409, 46-52. [CrossRef] [PubMed]

2. O'brien, J.L. Optical quantum computing. Science 2007, 318, 1567-1570. [CrossRef] [PubMed]

3. O’brien, J.L.; Furusawa, A.; Vučković, J. Photonic quantum technologies. Nat. Photonics 2009, 3, 687-695. [CrossRef]

4. Kennard, J.E.; Hadden, J.P.; Marseglia, L.; Aharonovich, I.; Castelletto, S.; Patton, B.R.; Politi, A.; Matthews, J.C.F.; Sinclair, A.G.; Gibson, B.C.; et al. On-chip manipulation of single photons from a diamond defect. Phys. Rev. Lett. 2013, 111, 213603. [CrossRef] [PubMed]

5. Schwagmann, A.; Kalliakos, S.; Farrer, I.; Griffiths, J.P.; Jones, G.A.; Ritchie, D.A.; Shields, A.J. On-chip single photon emission from an integrated semiconductor quantum dot into a photonic crystal waveguide. Appl. Phys. Lett. 2011, 99, 261108. [CrossRef]

6. Tonndorf, P.; Del Pozo-Zamudio, O.; Gruhler, N.; Kern, J.; Schmidt, R.; Dmitriev, A.I.; Bratschitsch, R. On-chip waveguide coupling of a layered semiconductor single-photon source. Nano Lett. 2017, 17, 5446-5451. [CrossRef]

7. Khasminskaya, S.; Pyatkov, F.; Słowik, K.; Ferrari, S.; Kahl, O.; Kovalyuk, V.; Rath, P.; Vetter, A.; Hennrich, F.; Kappes, M.M.; et al. Fully integrated quantum photonic circuit with an electrically driven light source. Nat. Photonics 2016, 10, 727-732. [CrossRef]

8. Rengstl, U.; Schwartz, M.; Herzog, T.; Hargart, F.; Paul, M.; Portalupi, S.L.; Jetter, M.; Michler, P. On-chip beamsplitter operation on single photons from quasi-resonantly excited quantum dots embedded in GaAs rib waveguides. Appl. Phys. Lett. 2015, 107, 021101. [CrossRef]

9. Senellart, P.; Solomon, G.; White, A. High-performance semiconductor quantum-dot single-photon sources. Nat. Nanotechnol. 2017, 12, 1026. [CrossRef]

10. Gazzano, O.; Michaelis de Vasconcellos, S.; Arnold, C.; Nowak, A.; Galopin, E.; Sagnes, I.; Lanco, L.; Lemaitre, A.; Senellart, P. Bright solid-state sources of indistinguishable single photons. Nat. Commun. 2013, 4, 1-6. [CrossRef]

11. Dousse, A.; Suffczynski, J.; Beveratos, A.; Krebs, O.; Lemaitre, A.; Sagnes, I.; Bloch, J.; Voisin, P.; Senellart, P. Ultrabright source of entangled photon pairs. Nature 2010, 466, 217-220. [CrossRef] [PubMed]

12. Kim, J.H.; Aghaeimeibodi, S.; Richardson, C.J.K.; Leavitt, R.P.; Englund, D.; Waks, E. Hybrid integration of solid-state quantum emitters on a silicon photonic chip. Nano Lett. 2017, 17, 7394-7400. [CrossRef] [PubMed]

13. Davanco, M.; Liu, J.; Sapienza, L.; Zhang, C.Z.; De Miranda Cardoso, J.V.; Verma, V.; Mirin, R.; Nam, S.W.; Liu, L.; Srinivasan, K. Heterogeneous integration for on-chip quantum photonic circuits with single quantum dot devices. Nat. Commun. 2017, 8, 889. [CrossRef] [PubMed]

14. Elshaari, A.W.; Zadeh, I.E.; Fognini, A.; Reimer, M.E.; Dalacu, D.; Poole, P.J.; Zwiller, V.; Jons, K.D. On-chip single photon filtering and multiplexing in hybrid quantum photonic circuits. Nat. Commun. 2017, 8, 379. [CrossRef] [PubMed]

15. Aghaeimeibodi, S.; Desiatov, B.; Kim, J.H.; Lee, C.M.; Buyukkaya, M.A.; Karasahin, A.; Richardson, C.J.K.; Leavitt, R.P.; Loncar, M.; Waks, E. Integration of quantum dots with lithium niobate photonics. Appl. Phys. Lett. 2018, 113, 221102. [CrossRef]

16. Cardenas, J.; Poitras, C.B.; Luke, K.; Luo, L.W.; Morton, P.A.; Lipson, M. High coupling efficiency etched facet tapers in silicon waveguides. IEEE Photonics Technol. Lett. 2014, 26, 2380-2382. [CrossRef]

17. Ates, S.; Agha, I.; Gulinatti, A.; Rech, I.; Badolato, A.; Srinivasan, K. Improving the performance of bright quantum dot single photon sources using temporal filtering via amplitude modulation. Sci. Rep. 2013, 3, 1397. [CrossRef]

18. Dolores-Calzadilla, V.; Romeira, B.; Pagliano, F.; Birindelli, S.; Higuera-Rodriguez, A.; Van Veldhoven, P.J.; Smit, M.K.; Fiore, A.; Heiss, D. Waveguide-coupled nanopillar metal-cavity light-emitting diodes on silicon. Nat. Commun. 2017, 8, 1-8.

19. Makhonin, M.N.; Dixon, J.E.; Coles, R.J.; Royall, B.; Luxmoore, I.J.; Clarke, E.; Hugues, M.; Skolnick, M.S.; Fox, A.M. Waveguide coupled resonance fluorescence from on-chip quantum emitter. Nano Lett. 2014, 14, 6997-7002. [CrossRef]

20. Prtljaga, N.; Coles, R.J.; O'Hara, J.; Royall, B.; Clarke, E.; Fox, A.M.; Skolnick, M.S. Monolithic integration of a quantum emitter with a compact on-chip beam-splitter. Appl. Phys. Lett. 2014, 104, 231107. [CrossRef]

21. Prtljaga, N.; Bentham, C.; O’Hara, J.; Royall, B.; Clarke, E.; Wilson, L.R.; Skolnick, M.S.; Fox, A.M. On-chip interference of single photons from an embedded quantum dot and an external laser. Appl. Phys. Lett. 2016, 108, 251101. [CrossRef]

22. Schnauber, P.; Schall, J.; Bounouar, S.; Höhne, T.; Park, S.I.; Ryu, G.H.; Heindel, T.; Burger, S.; Song, J.D.; Rodt, S.; et al. Deterministic integration of quantum dots into on-chip multimode interference beamsplitters using in situ electron beam lithography. Nano Lett. 2018, 18, 2336-2342. [CrossRef] [PubMed]

23. Mrowinński, P.; Schnauber, P.; Gutsche, P.; Kaganskiy, A.; Schall, J.; Burger, S.; Rodt, S.; Reitzenstein, S. Directional emission of a deterministically fabricated quantum dot-bragg reflection multimode waveguide system. ACS Photonics 2019, 6, 2231-2237. [CrossRef]

24. Dusanowski, Ł.; Köck, D.; Shin, E.; Kwon, S.H.; Schneider, C.; Höfling, S. Purcell-enhanced and indistinguishable single-photon generation from quantum dots coupled to on-chip integrated ring resonators. Nano Lett. 2020, 20, 6357-6363. [CrossRef]

25. Faraon, A.; Fushman, I.; Englund, D.; Stoltz, N.; Petroff, P.; Vučković, J. Dipole induced transparency in waveguide coupled photonic crystal cavities. Opt. Express 2008, 16, 12154-12162. [CrossRef]

26. Bentham, C.; Hallett, D.; Prtljaga, N.; Royall, B.; Vaitiekus, D.; Coles, R.J.; Clarke, E.; Fox, A.M.; Skolnick, M.S.; Itskevich, I.E.; et al. Single-photon electroluminescence for on-chip quantum networks. Appl. Phys. Lett. 2016, 109, 161101. [CrossRef]

27. Huo, Y.H.; Witek, B.J.; Kumar, S.; Cardenas, J.R.; Zhang, J.X.; Akopian, N.; Singh, R.; Zallo, E.; Grifone, R.; Kriegner, D.; et al. A light-hole exciton in a quantum dot. Nat. Phys. 2014, 10, 46-51. [CrossRef] 
28. Huo, Y.H.; Rastelli, A.; Schmidt, O.G. Ultra-small excitonic fine structure splitting in highly symmetric quantum dots on GaAs (001) substrate. Appl. Phys. Lett. 2013, 102, 152105. [CrossRef]

29. Aspnes, D.E.; Kelso, S.M.; Logan, R.A.; Bhat, R. Optical properties of AlxGa1- xAs. J. Appl. Phys. 1986, 60, 754-767. [CrossRef]

30. Basché, T.; Moerner, W.E.; Orrit, M.; Talon, H. Photon antibunching in the fluorescence of a single dye molecule trapped in a solid. Phys. Rev. Lett. 1992, 69, 1516. [CrossRef]

31. Dietrich, C.P.; Fiore, A.; Thompson, M.G.; Kamp, M.; Höfling, S. GaAs integrated quantum photonics: Towards compact and multi-functional quantum photonic integrated circuits. Laser Photonics Rev. 2016, 10, 870-894. [CrossRef]

32. Murray, E.; Ellis, D.J.P.; Meany, T.; Floether, F.F.; Lee, J.P.; Griffiths, J.P.; Jones, G.A.C.; Farrer, I.; Ritchie, D.A.; Bennett, A.J.; et al. Quantum photonics hybrid integration platform. Appl. Phys. Lett. 2015, 107, 171108. [CrossRef]

33. Wan, N.H.; Lu, T.J.; Chen, K.C.; Walsh, M.P.; Trusheim, M.E.; De Santis, L.; Bersin, E.A.; Harris, I.B.; Mouradian, S.L.; Christen, I.R.; et al. Large-scale integration of artificial atoms in hybrid photonic circuits. Nature 2020, 583, 226-231. [CrossRef]

34. Lukin, D.M.; Dory, C.; Guidry, M.A.; Yang, K.Y.; Mishra, S.D.; Trivedi, R.; Radulaski, M.; Sun, S.; Vercruysse, D.; Ahn, G.H.; et al. 4H-silicon-carbide-on-insulator for integrated quantum and nonlinear photonics. Nat. Photonics 2020, 14, 330-334. [CrossRef]

35. Vogel, M.M.; Ulrich, S.M.; Hafenbrak, R.; Michler, P.; Wang, L.; Rastelli, A.; Schmidt, O.G. Influence of lateral electric fields on multiexcitonic transitions and fine structure of single quantum dots. Appl. Phys. Lett. 2007, 91, 051904. [CrossRef]

36. Gerardot, B.D.; Seidl, S.; Dalgarno, P.A.; Warburton, R.J.; Granados, D.; Garcia, J.M.; Kowalik, K.; Krebs, O.; Karrai, K.; Badolato, A.; et al. Manipulating exciton fine structure in quantum dots with a lateral electric field. Appl. Phys. Lett. 2007, 90, 041101. [CrossRef] 